\title{
Bioabsorbable Vascular Scaffolds Eluting with Everolimus: for Percutaneous Coronary Intervention of Patients with de-novo Coronary Artery Lesion: Our Experiences at a Tertiary Hospital
}

\author{
AHMW Islam, S Munwar, S Talukder, AQM Reza, T Ahmed, AH Bhuiyan, R Masud, SR Sohel, \\ MS Alam, A Miah, S Chakrabarty, A Karim, AB Siddique \\ Dept. of Invasive and Interventional cardiology, Apollo Hospitals Dhaka
}

Key Words:

Bioabsorbable

vascular

scaffolds (BVS),

Drug eluting

stent (DES)

\begin{abstract}
:
Background: Aim of the study was to evaluate the primary procedural success of percutaneous coronary intervention (PCI) of de-novo coronary artery lesion by using Bioabsorbable Vascular Scaffold (BVS) ABSORB stents eluting Everolimus.

Methods: Total 16 patients were enrolled in this very preliminary study of BVS absorb. Among them, Male: 11 and Female: 5 . Total 20 stents were deployed. Mean age were for Male: 56 yrs, for Female: 60 yrs. Associated coronary artery disease (CAD) risk factors were Dyslipidemia, High Blood pressure, Diabetes Mellitus, Positive family history (FH) for CAD and Smoking. Patients were followed up clinically.

Results: Among the study group; 13 (81\%) were Dyslipidemic, 10(62.5\%) were hypertensive; 6 (37.5\%) patients were Diabetic, FH 3(18.75\%), and 2(18\%) were all male smoker. Female patients were more obese [Body Mass Index (BMI) M 25: F 27] and developed CAD in advance age. A common stented territory was for left anterior descending artery (LAD): 6 (37.5\%), left circumflex artery (LCX) 5 (25\%), right coronary artery (RCA) 6(37.5\%). One patient had both LCX and LAD stenting. Total 3 patients had double/overlapping stent in RCA lesion. Territory wise distributions of BVS ABSORB stent were for LAD 6(30\%), RCA 9 (45\%), and LCX 5 (25\%). There was no periprocedural or postprocedural complication.
\end{abstract}

Conclusion: BVS ABSORB Everolimus eluting vascular scaffold showed favorable clinical outcome without any major cardiac events (acute or late stent thrombosis, MI or death) over a period of 9 month. Thus, BVS ABSORB would be favorable alternative to other available drug eluting metallic stents.

(Cardiovasc. j. 2014; 6(2): 122-126)

\section{Introduction:}

The invention of balloon angioplasty as a Percutaneous treatment for obstructive coronary disease by Andreas Gruntzig in 1977 was a huge leap forward in cardiovascular medicine and undoubtedly, will always be remembered as a revolution in the field of revascularization. ${ }^{1}$ However, this technique was plagued by multiple problems, including the risk of acute vessel closure due to occlusive coronary dissection or acute or late stent thrombosis. ${ }^{2-4}$ The advent of bare metal stenting (BMS) and the landmark BENSTENT ${ }^{5}$ and STRESS 6 trials have established that the BMS provided a solution to acute vessel occlusion by sealing the dissection flaps and prevent vessel recoil. Later on, In the
RAVEL study, the drug eluting stent (DES) showed the reduction of neointimal hyperplasia and thus, stent restenosis. ${ }^{7}$ All these enthusiasms has been tempered by the wide spread concerns of, about the increased risk of late and very late stent thrombosis, ${ }^{8}$ abnormal vasoconstriction to acetylcholine distal to deployed stent. ${ }^{9,10}$

Recently, the invention and clinical application of BVS absorb vascular scaffold in human has established as an alternative to all available DES. BVS stent has a bioabsorbable polylactic acid with a bioabsorbable polylactic acid coating, that contain the antiproliferative drug everolimus. At 2 years, the stent struts were fully absorbed with complete integration of the scaffold into

Address of Correspondence : Dr. A H M Waliul Islam, Departmentt of Interventional and Invasive cardiology, Apollo Hospitals Dhaka, Bangladesh. Email-ahmwislam@apollodhaka.com. 
vessel wall and infiltration by smooth muscle cell (SMC). Thus, a bioabsorbable stent might have less potential for late stent thrombosis than a drug eluting stent because there will be no foreign material exposed to blood if endothelization delayed or incomplete than a drug eluting metallic stent. ${ }^{11}$

Since, its introduction, the uses of BVS with its potential effects in reducing restenosis with maintaining the integrity of vessel has been well established. To see the clinical outcome of the BVS ABSORB vascular scaffold in treating denovo coronary artery stenosis, we have carried out this prospective cohort study and followed up by noninvasive clinical assessment in our cardiac outpatients department.

\section{Methods:}

Study design: Uses of BVS was approved by the local ethics committee, and all patients gave written consent after briefing in detail about the BVS stent. In brief, patients were eligible for the study if they had single de novo native coronary lesions that could be covered with a single BVS and were included in the study. Patient were ineligible if the had evolving myocardial infarction, left main coronary artery lesion, an ostial lesion, a bifurcation lesion, a totally occluded lesion, a lesion with moderate to heavy calcification and were excluded from the study. Patients were followed up clinically at 6-8 weeks interval.

Study Population: Total 16 patients were enrolled in this very preliminary study. Among them, Male: 11 and Female: 6. Total 20 stents were deployed. Mean age were for Male: $59 \mathrm{yrs}$, for Female: 62 yrs. Associated CAD risk factors were Dyslipidemia, High Blood pressure, Diabetes Mellitus, Positive FH for CAD and Smoking (all male).

Study Device: The polymeric device consist of a backbone of poly-L-Lactide (PLLA) coated with poly-D, L-lactide (PDLLA) that contains and control the release of antiproliferative drug, Everolimus. The long chain of PLLA and PDLLA are progressively shortened as ester bonds between lactide repeat units are hydrolyzed. Ultimately, degrade to lactic acid and metabolized by Krebs cycle.

Stenting Procedure: CAG was performed as per standard protocol. Individual discretion was applied as per operator's choice, depending on patient's problem. Fully absorbable Everolimus eluting BVS scaffold (Abbott Vascular, Santa Clara, CA, USA) was used for PCI of de-novo coronary lesion. Coronary angioplasty was performed by implanting BVS scaffold following a mandatory Predilatation. Further stent apposition was done by gradual post dilatation. A successful procedures was defined as TIMI-3 antegrade flow, and $<20 \%$ residual stenosis in two orthogonal views.

Drug Therapy: All the patients received Aspirin $300 \mathrm{mg} /$ day and Clopidegrol as a loading dose $300 \mathrm{mg}$ prior to PCI, kept on for initial 9-12 months and advised to continue up till next 24 month. Patient also received atorovastatin along with standard Medical management for CAD. During the procedure, an intravenous heparin bolus (100IU/Kg) administered and the use GP $\mathrm{IIb} / \mathrm{III}$ receptor blocker was as per individual operator discretion. Quantitative angiographic measurements of the target lesion were obtained in order to deploy correct size stent.

Safety measure: All patients were followed up at a regular interval of $6-8 \mathrm{wks}$ in our cardiac outpatient department. To ensure the safety and efficacy of the devices, all patients were considered to assess clinically for the development of chest pain, hypotension, heart failure or Myocardial Infarction. In the event, if any, postprocedural CK-MB, ECG and Echo were advised.

Statistical analysis: Data were presented as mean $\pm \mathrm{SD}$ with percentage..

\section{Results:}

Table I. Shows the profile of studied population. Female patients were more obese (BMI; M 25: F 27) and developed CAD in advance age (M 56: $\mathrm{F}$ 60). CAD risk factors were more in male than female.

Table-I

Demographic Profile of patient

\begin{tabular}{lcc}
\hline & Male & Female \\
\hline Number & 11 & 5 \\
Age $(\mathrm{yrs})$ & $56 \pm 12$ & $60 \pm 7.6$ \\
BMI $\left(\mathrm{kg} / \mathrm{m}^{2}\right)$ & $25.5 \pm 2.0$ & $27.4 \pm 0.9$ \\
SBP $(\mathrm{mmHg})$ & $124.0 \pm 9.2$ & $124.0 \pm 11.4$ \\
DBP $(\mathrm{mmHg})$ & $74.5 \pm 5.2$ & $80.0 \pm 10.0$ \\
No. Risk Factor & $2.1 \pm 1.0$ & $2.2 \pm 0.4$ \\
RBS & $7.2 \pm 1.3$ & $9.5 \pm 4.5$ \\
Percentage of Lesion & $83.0 \pm 7.9$ & $81.0 \pm 7.4$ \\
\hline
\end{tabular}

Data were presented as Mean \pm SD 


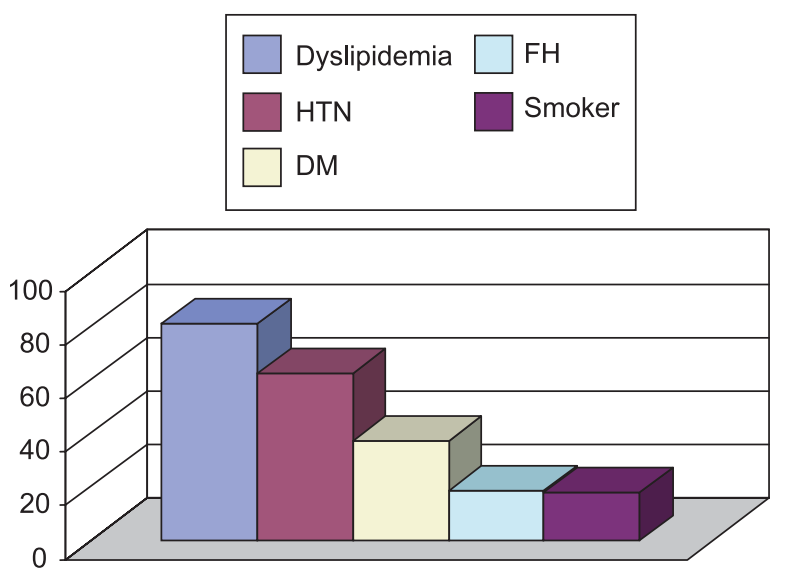

Fig.-1: Percentage distribution of CAD risk factors

Table II. Shows the average size of the BVS vascular scaffold used. Fig 1. shows the CAD risk factors. Among them, 13 (81\%) were Dyslipidemic, 10(62.5\%) were hypertensive; 6 (37.5\%) patients were Diabetic, FH 3(18.75\%), and 2(18\%) were all male smoker.. Fig 2. Territory wise distribution of BVS Absorb stent, were for: LAD 6(30\%), RCA 9 (45\%), and LCX 5 (25\%). Fig 3. Common stented territory were for: LAD 6 (37.5\%), LCX 5 (25\%), RCA 6(37.5\%).

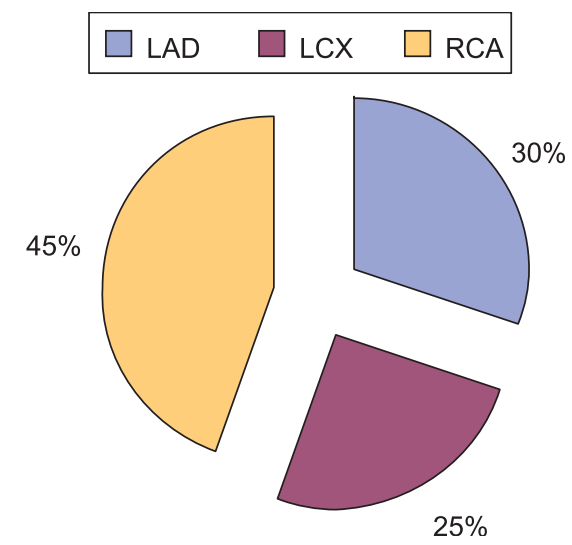

Fig.-2: Percentage distribution of coronary stents

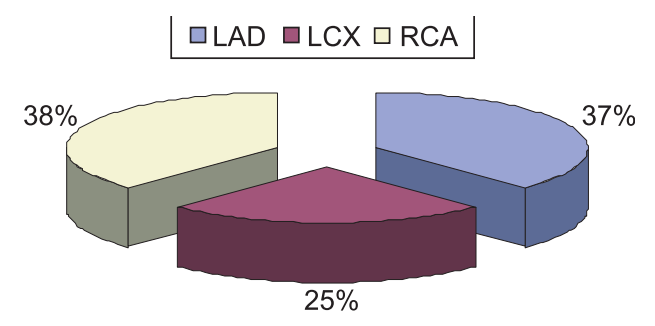

Fig.-3: Percentage distribution of lesion
Table-II

Average size of Stent used with inflation pressure

\begin{tabular}{lccc}
\hline & $\begin{array}{c}\text { Length } \\
(\mathrm{mm})\end{array}$ & $\begin{array}{c}\text { Diameter } \\
(\mathrm{mm})\end{array}$ & $\begin{array}{c}\text { Inflation } \\
\text { Pressure }(\mathrm{ATM})\end{array}$ \\
\hline LAD & $24.7 \pm 5.2$ & $2.8 \pm 0.25$ & $14.3 \pm 3.7$ \\
RCA & $31.0 \pm 16.6$ & $3.0 \pm 0.4$ & $15.0 \pm 2.75$ \\
LCX & $20.0 \pm 4.5$ & $2.8 \pm 0.3$ & $14.4 \pm 3.6$ \\
\hline
\end{tabular}

Data were presented as Mean \pm SD

\section{Discussion:}

In this non-randomized prospective cohort, all the patients were treated with BVS Absorb vascular scaffold eluting with Everolimus and followed up clinically on regular interval in the cardiac outpatient department. In this very preliminary study, only simple lesion were selected and treated with a BVS stent after predilatation of the culprit lesion. Complex lesion, CTO lesion and calcific lesion were excluded from the protocol. Patients were followed up by noninvasive means in the cardiac outpatient department of this hospital.

PTCA has been established as an alternative to coronary artery bypass surgery to treat selected patients with coronary artery disease. However, success of PTCA is limited by acute vessel occlusion and late restenosis. Stents were developed to overcome these issues, and first clinical application with metallic stent was conducted in $1986 .{ }^{3}$ All currently available stents, they induce varying degree of thrombogenesis and significant intimal hyperplasia. Late thrombosis may be another potential risk.

Stent malposition in cases of plaque shrinkage or positive vessel remodeling can also occur. Furthermore, metallic stent could preclude surgical revascularization and jail-sided branches, prevent expansive remodeling, eliminate reactive vasomotion and impairing the non-invasive imaging of coronaries by $\mathrm{CT}$ and MRI. Restenosis commonly occur within 3 to 6 months after coronary intervention. ${ }^{10}$ Therefore; the clinical need for stent scaffolding is limited after this period. Considering the short term need and the potential for long-term complications with metallic stents, stents made of biodegradable materials may be an ideal alternative. 
Tamai et al, ${ }^{15}$ demonstrated that the PLLA stents do not showed any stent thrombosis or early stent recoil. And at 6 month follow up all patients showed acceptable restenosis and target lesion revascularization, and no MI or CABG were recorded. Thus PLLA stents can be an attractive alternative to metallic stents and serve as a useful vehicle for locally administered drugs. Although, the first generation of BVS showed a slightly higher acute recoil and at 6 months an $11.8 \%$ reduction of in scaffold area and a $24.3 \%$ decrease in minimal luminal area were documented and dubbed late recoil. ${ }^{12,13}$ To minimize these recoil, the BVS revision 1.1 was tested in 101 patients of the ABSORB cohort B study and demonstrated that modified manufacturing process of the polymer and geometric changes in the polymorphic platform have substantially improved the performance to become comparable with current DES. ${ }^{14}$ Because of the biodegradable feature, longer-term follow up with multiple comparative study necessary to prove its superiority over the available metallic DES. In this regard, more patients need to include in the registry and to do randomize study to determine its safety and efficacy of PLLA stent coated with Everolimus in our population.

Several on going trials of BVS Absorb are promising, Tamai, et al, ${ }^{15,16}$ showed long term results similar to those of after BMS implantation, leading support to the feasibility and safety of a bioabsorbable PLLA polymer stent. The absorb trial ${ }^{17}$ showed, the overall $16.8 \%$ reduction of the luminal area at 6 months due to a mild shrinkage of the stent area. The only major adverse cardiac event was a non- $Q$ MI in one patient. After 2 years the biodegradable stent was incorporated into the vessel wall with the documented restoration of vasomotion suggest healing of the artery. In addition, it appeared that the vessel lumen enlarged and the plaque/media diminished. ${ }^{18}$ The 3 year clinical outcome is quite encouraging: IDMACE is $3.4 \%$ without any late complication. Since, the first generation BVS Everolimus stents showed signs of shrinkage at 6 months, modified manufacturing process of the polymer and geometric changes in the polymeric platform substantially improved the medium term performance of this new generation drug eluting scaffold to be comparable to those with currently available DES. ${ }^{14}$ Thus, the absence of a foreign body and restoration of vasomotion raise the hope of abnormal healed vessel that could be without the risk of thrombosis.

In our present perspective of non-randomized cohort, total 20 stents were deployed in 17 territories of total 16 patients. 3 patients has overlapped stented segment. One patient has double vessel stent. Large size BVS scaffold was used in RCA. Because of the small number of patient, it was very difficult to address whether BVS ABSORB vascular scaffold eluted with the drug everolimus is superior over the other available metallic DES. No stent fracture during the deployment and acute or sub-acute stent restenosis occurred in the study. Careful consecutive predilatation of the culprit lesion before the stent deployment was mandated and postdilatation was done to ensure better stent apposition.

We need more patient inclusion, randomization into PCI with BVS absorb and with DES metallic stents to compare the superiority of PCI of BVS ABSORB. In addition, we need to address the superiority of BVS absorb Everolimus drug eluting stents over DES metal stents in treating coronary stenosis. In this regard, we need multicenter study in perspective of Bangladeshi patient population. We need to address the impact of CAD risk factor, overlapped stent, dual anti-platelet therapy (DAPT) has any role in inducing stent thrombosis and the development of instent restenosis in the patient treated with BVS vascular scaffold.

Study Limitations: We don't have intravascular ultrasonogram (IVUS) and Fractional Flow Reserve (FFR)

\section{Conclusion:}

BVS ABSORB Everolimus eluting vascular scaffold for treating de novo coronary artery stenosis in our patient perspective showed excellent clinical safety with no major adverse cardiac event at 9 month clinical follow up at our center. To address its superiority compare to other available drug eluting stent, we need more randomized comparative study.

Conflict of Interest - None. 


\section{References:}

1. Gruntzig AR, Senning A, Siegenthaler WE. Non-operative dilatation of coronary-artery stenosis: Percutaneous transluminal coronary angioplasty. $N$ eng $J$ Med 1979; 301:61-68

2. de Feyter PJ, de Jaegree PW, et al. Incidence, predictors and management of acute coronary occlusion after coronary angioplasty. Am Heart J 1994;127:643-651.

3. Sigwart U, Puel J, Mirkovitch V, et al. Intravascular stents to prevent occlusion and restenosis after transluminal angioplasty. $N$ Eng J Med 1987;316: 701-706.

4. Serruys PW, Luijiten HE, Beatt KJ, et al. Incidence of restenosis after successful coronary angioplasty: a time related phenomena: a q2uantritative angiographic study of 342 consecutive patients at 1,2,3 and 4 months. Circulation 1988; 77:361-371.

5. Serruys PW, de jaegere P, Kiemenji F, et al. A comparison of balloon expandable stent implantation with balloon angioplasty in patent with coronary artery disease: Benstent study group. N Eng J Med 1994; 331: 489-495.

6. Fischman DL, Leon MB, Baim DS, et al. A randomized comparison of coronary stent placement and balloon angioplasty in the treatment of coronary artery disease: stent restenosis study Investigators. N Eng J Med 1994; 331:496-501.

7. Morice MC, Serruys PW, Sousa JE, et al. A randomized comparison of a Sirolimus eluting stent with a standard stent for coronary revascularization. $N$ Eng J Med 2002;346:1773-1780.

8. Daem,on J, Wenaweser P, Tsuchida K, et al. Early and late coronary stent thrombosis of Sirolimus eluting and Paclitaxel eluting stents in routine clinical practice: data from a large two-institutional cohort study. Lancet 2007; 369:667-678.
9. Hofma SH, Van de Giessen WJ, et al. Indication of long term endothelial dysfunction after Sirolimus eluting stent implantation. Eur Heart J 2006; 27:166-170.

10. Togni M, Windecker S, Cocchia R, et al. Sirolimus eluting stents associated with paradoxic coronary vasoconstriction. J Am Coll Cardiol 2005; 46:231-236.

11. Kotani J, Awata M, Nanto S et al. Incomplete neointimal coverage of sirolimus eluting stents: angioscopic findings. J Am Coll Cardiol 2006; 47:2108-2111.

12. Tanimoto S, Serruys PW, Thuesen L, et al. Comparison of in vivo acute stent recoil between the bioabsorbable Everolimus eluting stent and Everolimus cobalt chromium stent; insights from absorb and SPIRIT Trial. Catheter Cardiovasc Interv 2007;70: 515-523.

13. Tanimoto S, Bruining N, Van Domburg RT, et al. Late stent recoil of the bioabsorbable Everolimus eluting stent and its relationship with plaque morphology. J Am Coll Cardiol 2008; 52:1616-1620.

14. Serruys PW, Onuma Y, Ormiston JA, et al. Evaluation of the second generation of a bioabsorbable Everolimus drug eluting vascular scaffold for the treatment of de-novo coronary artery stenosis. Circulation 2010; 122:2301-2312.

15. Tamai H, Igaki K, Kyo E, et al. Initial and 6 months result of biodegradable poly-lactic acid coronary stents in humans. Circulation 2000; 102:399-404

16. Tamai H. Biodegradable stents four year follow-up. Washington: TCT 2004

17. Ormiston JA, Serruys PW, Regar E, et al. A bioabsorbable Everolimus eluting coronary stent system for patients with single de novo coronary artery lesion (ABSORB): a prospective open-label trial. Lancet 2008; 371:899-907.

18. Serruys PW, Ormiston JA, Onuma Y, et al. A bioabsorbable Everolimus eluting coronary stent system (absorb): 2 year outcomes and results from multiple imaging methods. Lancet 2009; 373:897-910. 